\title{
Spinal cord lesions in Bangladesh: an epidemiological study 1994-1995
}

\author{
Md Fazlul Hoque*,1, Christoph Grangeon ${ }^{1}$ and Kylie Reed ${ }^{1}$ \\ ${ }^{1}$ Centre for the Rehabilitation of the Paralysed (CRP), Bangladesh
}

\begin{abstract}
Objectives: Spinal Cord Lesions are a major public health problem in Bangladesh. This epidemiological study was undertaken in order to identify the causes of spinal cord lesions and thus to allow prevention and control programs to be developed.

Materials and methods: The records of 247 patients with spinal cord lesions admitted to The Centre for the Rehabilitation of the Paralysed (CRP), Savar, Dhaka from January 1994 to June 1995 were reviewed retrospectively. Comparisons were made with the reports of studies from other countries, both developing and developed.

Results: The most common cause of traumatic lesions was a fall from a height followed by falling when carrying a heavy weight on the head and road traffic accidents. Most of the patients were between $20-40$ years old and the overall age group ranged from $10-70$ years. The male:female ratio was $7.5: 1.0$. Among the traumatic spinal cord lesions, $60 \%$ were paraplegics and $40 \%$ tetraplegics. Among the non-traumatic spinal cord lesions cases $84 \%$ were paraplegics and $16 \%$ tetraplegics. The leading cause of death resulted from respiratory complications and these deaths occurred in the very early period of admission.

Conclusion: From the results it can be deduced that the high incidence of spinal cord lesion as a result from falls from a height, and from falling when carrying a heavy weight on the head, can be explained by the mainly agricultural based economy of Bangladesh. The most common age group (10-40 years) of patients reflects the socio-economic conditions of Bangladesh. The male:female ratio $(7.5: 1.0)$ of patients with a spinal cord lesion is due to the socio-economic status and to the traditional culture of the society.
\end{abstract}

Keywords: spinal cord lesion; epidemiological; traumatic; non-traumatic; paraplegic; tetraplegic

\section{Introduction}

Injuries and diseases affecting the spinal cord and complicated by neurological damage are an important health problem in Bangladesh as they carry high rates of morbidity and mortality. Intensive medical care which must include rehabilitation is essential, but even so the results of spinal cord damage have a major economic and social impact both on the patient's family and on society in general. An increased understanding of the relevant aetiological factors should encourage the introduction of better methods of prevention.

It is of interest that a differing incidence of the causes of spinal cord lesions in countries at various stages of development has been revealed by recent studies, ${ }^{1-3}$ and it is for this reason that we have reviewed retrospectively a consecutive series of patients admitted to the Centre for the Rehabilitation of the Paralysed, (CRP), in Bangladesh. The CRP is a non-governmental organization specializing in the management of patients with spinal cord lesions. It

*Correspondence: Md Fazlul Hoque, Consultant Orthopaedic Surgeon, Centre for the Rehabilitation of the Paralysed (CRP), PO-CRP, Chapain, Savar, Dhaka, Bangladesh has 100 beds, and it provides free services to all the people of Bangladesh. Management is based on a multi- and inter-disciplinary approach, with emphasis on the development of community based rehabilitation programs. There are three full-time doctors on the staff, and regular voluntary visits are made by two senior neurosurgeons. There is a full team of nursing staff, nursing aids, physiotherapists, occupational therapists and social workers all of whom are supported by short term volunteers from home and abroad.

It is possible that our results may be influenced by the fact that the CRP receives many patients at a late stage from all areas of the country, and often after they have received their initial treatment. This may explain some of the differences between our results and those recorded from other Asian countries. ${ }^{1-3}$

\section{Methods}

The records of all patients with a spinal cord lesion who were admitted (for the first time) to the CRP in the 18 months between January 1994 and June 1995 were studied retrospectively. 
Spinal cord injury was defined according to Krauss et $a l^{4}$ The American Spinal Injury Association's Classification was used for recording functional disability. ${ }^{5}$

\section{Results}

The records of 247 patients included 218 males (88\%) and 29 females $(12 \%)$, giving a male to female ratio of $7.5: 1.0$. Most of the patients were aged between 10 and 40 years, with $19 \%$ between $10-20$ years, $42 \%$ $20-30$ years, $20 \% 30-40$ years, $15 \% 40-50$ years and $4 \% 50-60$ years.

\section{Hospital stay and mortality}

The average length of stay in hospital was about 5 months with a spread from 1-6 months and was greater for those who arrived with pressure sores (Figure 1). Figure 1 illustrates that of patients with pressure sores, $(34 \%)$ remain in hospital more than 5 months in comparison to $(14 \%)$ of patients without pressure sores. This highlights that pressure sores are a major factor in delayed rehabilitation, and therefore reduce the overall number of patients who can be treated at CRP. Eighteen patients died $(7 \%$ of admissions).

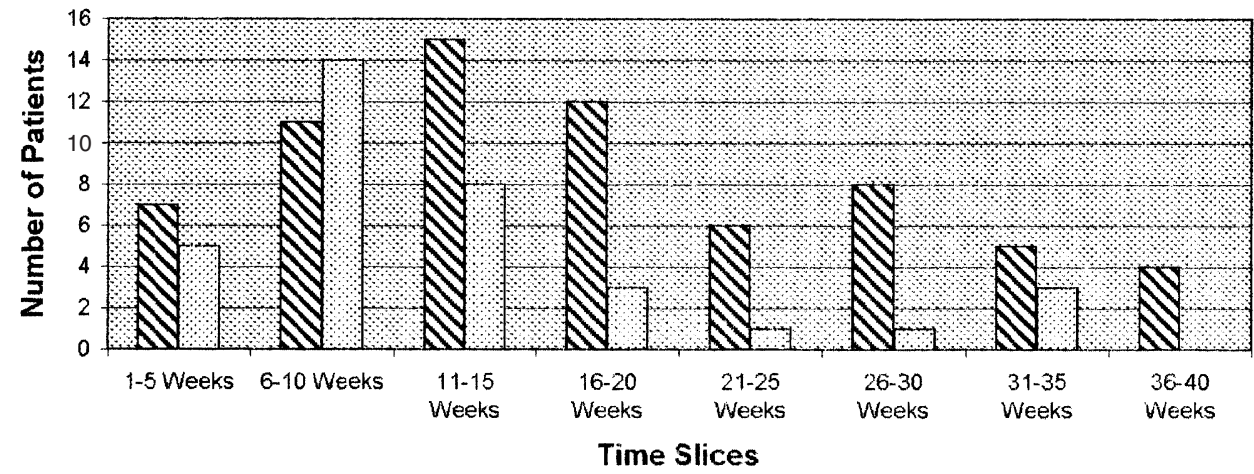

Q Fatient with Pressure sore [ Patient without Pressure sore

Figure 1 Length of stay at the Hospital

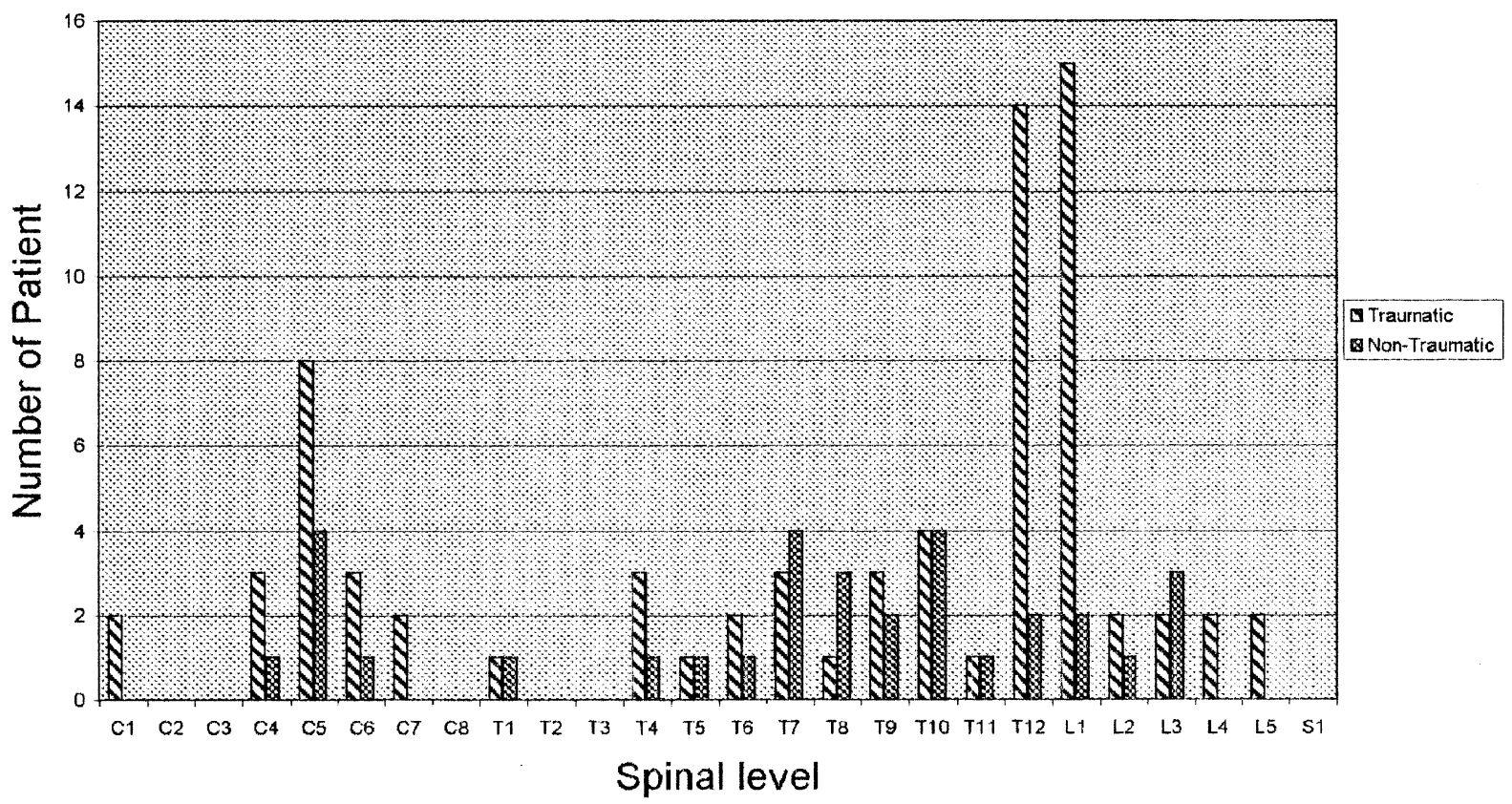

Figure 2 Distribution according to level of traumatic and non-traumatic lesions 


\section{Aetiology}

The spinal cord lesions were considered to be either 'traumatic' or 'non-traumatic'. Among the 179 'trauma' patients $(72 \%)$, there were three main causes of injury. Seventy-six $(43 \%)$ resulted from a fall from a height (such as a tree). Thirty-seven injuries $(20 \%)$ were associated with falling while carrying a heavy load on the head (a common practice in Bangladesh). Thirty-three $(18 \%)$ were a result of a road traffic accident. Eleven patients $(6 \%)$ formed a very diverse group which included assault, stab injury, sports injury and bull attack. There was insufficient data available for 22 patients $(13 \%)$. Of the patients in this 'traumatic' group 107 were paraplegic $(60 \%)$, and 72 tetraplegic $(40 \%)$.

Among the 68 patients $(28 \%)$ in the 'non-traumatic' spinal cord lesion group the main cause was Pott's disease (19 patients $28 \%$ ), followed by 14 patients with a tumour $(21 \%)$, seven cases of transverse myelitis $(10 \%)$, six from a prolapsed intra-vertebral disc $(9 \%)$, four with Guillain Barre syndrome $(6 \%)$ and one associated with cervical spondylosis $(1 \%)$. There were $17(25 \%)$ cases which remained undiagnosed. In this 'non-traumatic' group there were $57(84 \%)$ paraplegic and $11(16 \%)$ tetraplegic.

\section{Level of spinal cord lesion}

The level of the lesions is shown in Figure 2, and this reveals that the commonest problems were at the $\mathrm{C} 5$, and the T12/L1 levels for the 'traumatic' cases; and at C5, and between T7/L4 for the 'non-traumatic'. Pott's disease was responsible for lesions between T7-T12.

\section{Complications}

We studied the two main complications of Urinary Tract Infection (153 patients 62\%) and Pressure Sores (94 patients 38\%). Culture of the urine revealed three main organisms, Klebsiella in 41 patients $(29 \%)$, Proteus in $45(32 \%)$, and E. Coli in $56(39 \%)$.

Infection of the Pressure sores was with Staphylococcus in $23(52 \%)$, Proteus in $11(25 \%)$, and Klebsiella in $10(23 \%)$.

\section{Discussion}

Currently there is no National Spinal Cord Lesion Register in Bangladesh, and a survey of the available literature has not revealed any previous reports from this Country. Therefore, it is impossible to know or even to estimate the total number of patients with Spinal Cord Lesions in Bangladesh, as many never arrive at the CRP.

Our male to female ratio of $7.5: 1.0$ is higher in Japan and in Jordan, ${ }^{3,6}$ and this is not surprising in a country such as Bangladesh where the majority of women remain in the home as housewives, and are not subject to the more violent forms of accidents to which men are exposed. This supports the tenets of $\mathrm{Kuhn}^{7}$ who concluded that 'the sex ratio reflects the socioeconomic and cultural status of a society'.

The length of stay at the CRP averaged 5 months as we pursue an active policy of rehabilitation and early return home. However those patients who arrived with pressure sores stayed an average of 27 weeks while those without this problem were discharged after 14 weeks (Figure 1). Most of our patients with Spinal Cord Injuries were in the working group aged between $10-40$ years, and this is similar to that reported from Jordan and Thailand. ${ }^{2,6}$ However, in a more developed country such as Japan the average age is between $40-$ 70 , with a peak at 59 years. $^{3}$ It seems that our younger patients had fallen from a significant height, while the 'elderly' Japanese patients had fallen while on the ground. ${ }^{3}$

Falling from a height was the most common 'traumatic' cause of spinal cord injury in our series (76 patients, $43 \%$, and RTA $18 \%$ ), and this is similar to that reported from India (fall 55\%, and RTA 13\%), ${ }^{1}$ Nigeria (fall $48 \%$, and RTA $36 \%$ ) $^{8}$ and Romania (fall $59 \%$, and RTA $13 \%){ }^{9}$ In Chiang Mai, Thailand, falls accounted for $43 \% .{ }^{10}$ The large number of falls in Bangladesh is a result of fruit harvesting which is an important part of our largely agricultural economy. We also found in Bangladesh that the second main cause of spinal cord injury resulted from carrying heavy weights on the head. These injuries at the C5/C6 level occur when a person trips and falls while carrying a heavy load on his or her head. This usually results in a ligamentous lesion which is probably of a 'twisting' nature. The exact mechanics of these injuries merit further study. These accidents are more common in Bangladesh than elsewhere.

While road traffic accidents (RTA) are the main source of spinal cord injuries in more developed countries, in Bangladesh they are less often the cause (only 18\%) than a fall from a height, or a fall when carrying a heavy load on the head. Most of our RTA's involve passengers of 'three wheel vehicles' like 'baby taxis' and 'rickshaws'.

As is commonly accepted, the region of the fifth cervical vertebra, and the junction of the twelfth thoracic and first lumbar vertebrae were the segments most exposed to trauma. The non-traumatic cases commonly involved either C5-C7 or T7-L4. Pott's disease most often affected the region of $\mathrm{T} 7-\mathrm{T} 12$ and there were no examples of cervical tuberculous disease in this series.

It is possible that the relatively low mortality rate of $7 \%$ in our hospital is due to that fact that we rarely admit acute or fresh cases, as this is when many of these patients die.

We were not surprised that bacteria from the gastro-intestinal tract were responsible for urinary tract infections, and that pressure sores were invaded by skin organisms.

Thus, the results of this small retrospective study have shown some differences in the aetiology of spinal cord lesions seen in Bangladesh when compared with 
other countries in Asia. We propose to study the mechanism responsible for lesions of the cervical cord following a fall when carrying a heavy load on the head.

\section{Acknowledgements}

Thanks are due to Geoffrey Walker FRCS for help and advice in preparing the manuscript. This was typed by Maggie Muldoon to whom we are most grateful. Farhan Haque was responsible for the Figures and Tables.

\section{References}

1 Chacko V, Joseph B, Mohanty SP, Jacob T. Management of spinal cord injury in a general hospital in rural India. Paraplegia 1986; 24: $330-335$.
2 Parareya K. Traumatic spinal cord injury in Thailand: an epidemiological study in Sirijaj hospital, 1989-1994. Spinal Cord 1996; 34: 608-610.

3 Shingu $\mathrm{H}$ et al. A nation wide epidemiological survey of spinal cord injuries in Japan from January 1990 to December 1992. Spinal Cord 1995; 33: $183-188$.

4 Krauss JF et al. Incidence of traumatic spinal cord region. $J$ Chron Dis 1975; 25: $471-492$.

5 Ditunno JF, AA Young WH, Donovan WH, Creasy G. The International Standards Booklet for Neurological and functional classification of Spinal Cord injury. Paraplegia 1994; 32: 70 - 80 .

6 Otom A et al. Traumatic spinal cord injury in Jordan - an epidemiological study. Spinal Cord 1997; 35: $253-255$

$7 \mathrm{Kuhn} \mathrm{W}$ et al. Comparison of spinal cord injury in females and in males, 1979 - 1981 Basle. Paraplegia 1983; 21: 154-160.

8 Okonkwo CA. Spinal cord injuries in Enugu, Nigeria preventable accidents. Paraplegia 1988; 21: $12-18$.

9 Soopramanien A. Epidemiology of spinal injuries in Romania. Paraplegia 1994; 32: $715-722$.

10 Kovindha A. Spinal cord injuries in Maharaj Nakorn Chiang Mai Hospital: 5 years retrospectively. Chiang Mai Med Bull 1985; 24: $179-185$. 